\section{Closing in on the cause of insulin resistance and type 2 diabetes}

\author{
Matthew J. Brady and Alan R. Saltiel \\ Department of Cell Biology, Parke-Davis Pharmaceutical Research Division/Warner-Lambert \\ Company, Ann Arbor, Michigan 48105, USA
}

Address correspondence to: Alan R. Saltiel, 2800 Plymouth Road, Ann Arbor, Michigan 48105, USA. Phone: (734) 622-3960; Fax: (734) 622-5668; E-mail: Alan.Saltiel@wl.com.

Prospective epidemiological studies across several populations have indicated that insulin resistance may be the primary defect in the development of type 2 diabetes (DM2), as it can be detected long before the deterioration of glucose tolerance occurs. The continuum of the disease progresses as insulin resistance worsens, and frank diabetes appears when the $\beta$ cell can no longer compensate with increased insulin secretion. Thus, understanding the cellular events involved in insulin action, as well as the primary molecular site underlying insulin resistance, will have a major impact on efforts to develop new treatments for diabetes.

Insulin normally increases the uptake, storage, and oxidation of glucose in skeletal muscle. Insulin-stimulated glucose disposal is significantly reduced in insulin-resistant subjects, reflecting a defect in glucose transport, phosphorylation, and utilization or storage. Shulman and colleagues (1) have quantified glucose metabolism using nuclear magnetic resonance in patients with DM2,

Figure 1

Insulin stimulates glucose transport through multiple signaling pathways. Insulin causes the release of glucose storage vesicles (GSV) from intracellular tethering sites. Additionally, insulin stimulates the binding of the vesicular protein VAMP2 to syntaxin-4, allowing for vesicle docking at the plasma membrane. It is likely that insulin promotes GSV trafficking to the plasma membrane via a PI 3-kinase/Akt-dependent signaling cascade, but uses a PI 3-kinase-independent pathway to regulate vesicular fusion with the cell membrane. pinpointing glucose transport as the primary site at which insulin action fails. Because glucose uptake and metabolism are critical for the control of glycogen synthase activity by insulin, impaired glucose transport will also lead to a secondary reduction in glucose storage in muscle.

The stimulation of glucose transport by insulin involves the translocation of vesicles containing the GLUT4 glucose transporter isoform from intracellular sites to the cell surface (2). This is a multistep process involving the release of GLUT4 vesicles from their intracellular tethering sites, trafficking of the vesicles to the plasma membrane, and subsequent docking and fusion to expose the transporters at the cell surface. Thus, it is likely that the insulin receptor generates multiple signals to regulate this complex process. One critical pathway involves activation of phosphoinositide 3-kinase (PI 3kinase), which leads to the subsequent phosphorylation and activation of the protein kinase Akt (or protein kinase
B). While some studies have shown that Akt is essential in GLUT4 vesicle translocation, others have disputed this claim (reviewed in ref. 3). Moreover, several other growth factors and adhesion molecules can activate PI 3-kinase and Akt without increasing glucose transport. Thus, the exact requirement for Akt activation, both as an integral signaling component of insulin action on glucose metabolism and as a potential locus for development of insulin resistance and DM2, remains controversial.

GLUT4 levels are not reduced in diabetic patients (4), indicating that the defect lies in the signaling pathways or the membrane trafficking machinery. In this issue of the JCI, Kim et al. investigated the involvement of Akt in the development of insulin resistance and DM2 (5). The effects of insulin on glucose metabolism and enzymatic activation in obese, insulin-resistant, and diabetic patients were compared with the effects in lean controls. To our knowledge, the authors have demonstrated for the first time that Akt is phospho-

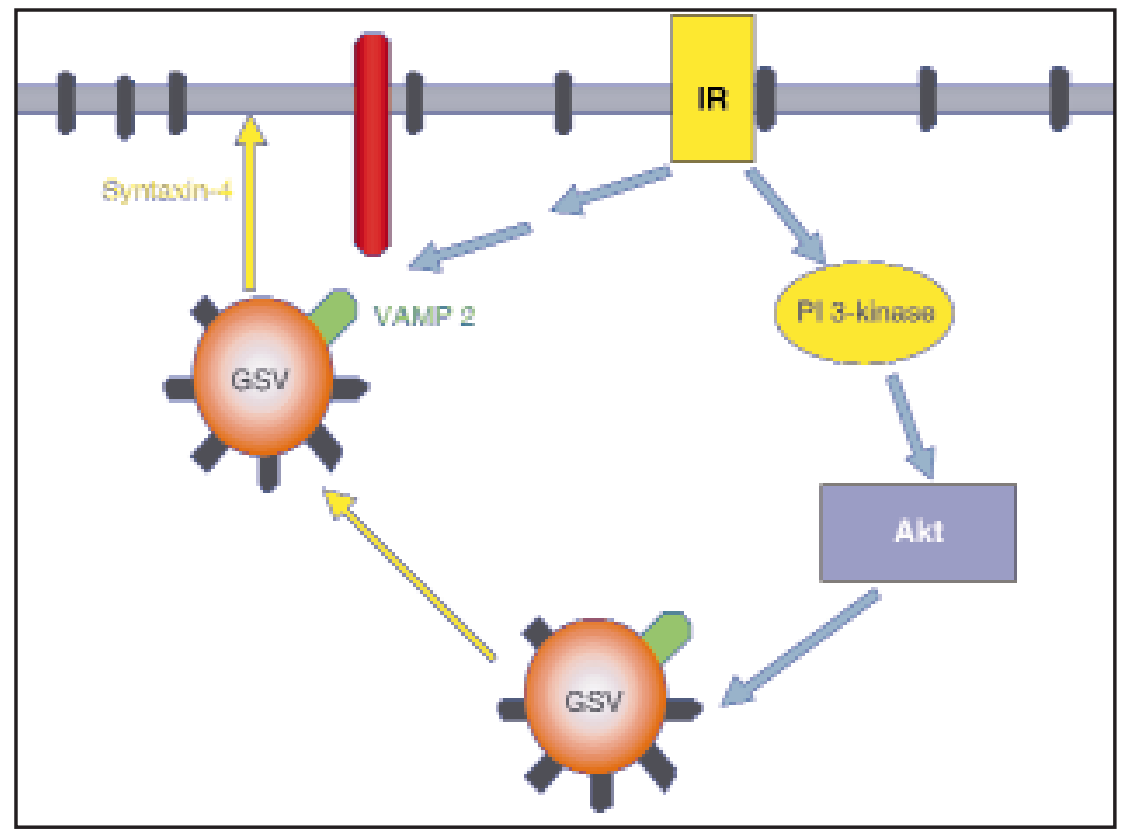


rylated and activated in vivo in response to insulin in human skeletal muscle biopsies. Surprisingly, the degree of phosphorylation and enzymatic activation was comparable in all 3 patient groups, despite the fact that the stimulation of glucose disposal, PI 3-kinase, and glycogen synthase activities was severely impaired in muscle from obese nondiabetic and diabetic subjects compared with controls. These results strongly suggest that Akt does not play a crucial role in the development of insulin resistance in human skeletal muscle, or in the progression to frank diabetes. However, these data do not preclude the involvement of Akt in the normal regulation of GLUT4 trafficking by insulin. Indeed, the degree of Akt activation correlated well with glucose disposal rate in the lean control group.

The cause of impaired GLUT4 translocation in insulin-resistant subjects is thus still unclear. One possibility is that the defect lies downstream of Akt, perhaps in the yet unidentified substrates of the kinase. Alternatively, PI 3-kinase activation may stimulate the activity of another kinase, such as $\mathrm{PKC}-\zeta$, which, in turn, can mediate insulin-stimulated glucose transport (6). However, there are also likely to be other signaling pathways involved in insulin-stimulated GLUT4 translocation. A cell-permeable derivative of $\mathrm{PIP}_{3}$, a lipid signaling product of PI 3-kinase, can increase GLUT4 translocation in cells pretreated with insulin and the PI 3-kinase inhibitor wortmannin (7). However, $\mathrm{PIP}_{3}$ is ineffective in the absence of insulin, indicating that at least one PI 3-kinase-indepedent pathway is required for GLUT4 mobilization. This result may explain the inability of other growth factors to increase glucose transport, despite their robust stimulation of PI 3-kinase and Akt.

The precise mechanisms by which GLUT4 vesicles are released from intracellular sites and subsequently fuse with the plasma membrane remain uncertain. The docking of GLUT4 vesicles at the cell membrane is mediated by the interaction of the vesicular $\mathrm{v}$-SNARE protein VAMP2 with membrane-associated t-SNARE proteins, such as syntaxin-4 and Munc18c (2). This interaction is also likely to be modulated by insulin, because of the dissociation of a regulatory protein that masks syntaxin-4, allowing for both the subsequent binding of VAMP2 and GLUT4 vesicle fusion (8). Interestingly, the regulation of SNARE interaction by insulin appears to be unaffected by PI 3-kinase inhibitors. It is tempting to speculate that PI 3-kinase activation by insulin mediates the release of GLUT4 vesicles from intracellular docking sites, whereas a second, PI 3-kinase-independent pathway regulates GLUT4 vesicle fusion with the plasma membrane (Figure 1).

The identification of defective glucose transport as the primary locus causing insulin resistance has been a major step forward, yet the specific molecule(s) that is compromised remains unclear. Although the patient pool was limited in size, and the results await confirmation in larger studies, the present work discounts the involvement of reduced Akt activation in the development of DM2. Clearly, attention will now be focused on molecules downstream of Akt activation, and on defining the players in the second signaling pathway, which may confer the unique ability of insulin to regulate glucose homeostasis.

1. Cline, G.W., et al. 1999. Impaired glucose transport as a cause of decreased insulin-stimulated muscle glycogen synthesis in type 2 diabetes. $N$. Engl. J. Med. 341:240-246.

2. Pessin, J.E., Thurmond, D.C., Elmendorf, J.S. Coker, K.J., and Okada, S. 1999. Molecular basis of insulin-stimulated GLUT4 vesicle trafficking. Location! Location! Location! J. Biol. Chem. 274:2593-2596

3. Czech, M.P., and Corvera, S. 1999. Signaling mechanisms that regulate glucose transport. $J$. Biol. Chem. 274:1865-1868.

4. Kahn, B.B. 1992. Facilitative glucose transporters: regulatory mechanisms and dysregulation in diabetes. J. Clin. Invest. 89:1367-1374.

5. Kim, Y.-B., Nikoulina, S., Ciaraldi, T.P., Henry, R.R., and Kahn, B.B. 1999. Normal insulindependent activation of Akt/protein kinase B, with diminished activation of phosphoinositide 3-kinase, in muscle in type 2 diabetes. J. Clin. Invest. 104:733-741.

6. Standaert, M.L., et al. 1997. Protein kinase C-zeta as a downstream effector of phosphatidylinositol 3-kinase during insulin stimulation in rat adipocytes. Potential role in glucose transport. $J$. Biol. Chem. 272:30075-30082.

7. Jiang, T., et al. 1998. Membrane-permeant esters of phosphatidylinositol 3,4,5-triphosphate. J. Biol. Chem. 273:11017-11024.

8. Min, J., et al. 1999. Synip: a novel insulin-regulated syntaxin 4 binding protein mediating GLUT4 translocation in adipocytes. Mol. Cell. 3:751-760. 